\title{
Burnout of Healthcare Workers in Acute Care Geriatric Facilities During the COVID-19 Crisis: An Online-Based Study
}

\author{
Mohamad El Haj $j^{\mathrm{a}, \mathrm{b}, \mathrm{c}, *}$, Philippe Allain ${ }^{\mathrm{d}, \mathrm{e}}$, Cédric Annweiler ${ }^{\mathrm{f}}$, Claire Boutoleau-Bretonnière ${ }^{\mathrm{g}}$, \\ Guillaume Chapelet $^{\mathrm{h}, \mathrm{i}}$, Karim Gallouj ${ }^{\mathrm{b}}$, Dimitrios Kapogiannis ${ }^{\mathrm{j}}$, Jean Roche ${ }^{\mathrm{k}}$ \\ and Abdel Halim Boudoukha ${ }^{\mathrm{a}}$ \\ ${ }^{a}$ Nantes Université, Univ Angers, Laboratoire de Psychologie des Pays de la Loire (LPPL - EA 4638), Nantes, \\ France \\ ${ }^{\mathrm{b}}$ Unité de Gériatrie, Centre Hospitalier de Tourcoing, Tourcoing, France \\ ${ }^{\mathrm{c}}$ Institut Universitaire de France, Paris, France \\ ${ }^{\mathrm{d}}$ Laboratoire de Psychologie des Pays de la Loire, LPPL EA 4638, SFR Confluences, UNIV Angers, Nantes \\ Université, Maison de la recherche Germaine Tillion, Angers Cedex 01, France \\ ${ }^{\mathrm{e}}$ Département de Neurologie, CHU Angers, Angers, France \\ ${ }^{\mathrm{f}}$ Department of Geriatric Medicine, Angers University Hospital, Angers, France \\ ${ }^{\mathrm{g}}$ CHU Nantes, Inserm CIC04, Département de Neurologie, Centre Mémoire de Ressources et Recherche, Nantes, \\ France \\ ${ }^{\mathrm{h}}$ Université de Nantes, Inserm, TENS, The Enteric Nervous System in Gut and Brain Diseases, IMAD, Nantes, \\ France \\ ${ }^{\mathrm{i}}$ CHU Nantes, Clinical Gerontology Department, Bd Jacques Monod, Nantes, France \\ ${ }^{\mathrm{j}}$ Laboratory of Clinical Investigation, National Institute on Aging, Baltimore, MD, USA \\ ${ }^{\mathrm{k}} \mathrm{CHU}$ de Lille, Department of Geriatric Psychiatry, Lille, France
}

Accepted 21 September 2021

\begin{abstract}
.
Background/Objective: The COVID-19 crisis has been increasing the burden of healthcare workers in acute care geriatric facilities. These workers have been dealing with drastic changes in the care they provide to their residents including cancelation of group activities and communal dining and even restrictions of activities outside rooms. Healthcare workers have also been devoting more time and energy to perform COVID-related medical duties. Geriatric facilities have been facing shortages in equipment and supplies, as well as staffing shortages. Finally, healthcare workers have been facing challenges regarding their personal safety and that of their families. Consequently, we hypothesized the presence of high levels of burnout among healthcare workers during the COVID-19 crisis.

Methods: To evaluate burnout in healthcare workers in French acute care geriatric facilities, we used an online survey based on the Oldenburg Burnout Inventory. Eighty-four healthcare workers answered the survey, during April of 2020.

Results: Analysis demonstrated that they were experiencing medium levels of burnout, exhaustion, and disengagement.

Conclusion: This level of burnout reflected their fatigue, loss of energy, and/or feelings of being overextended and exhausted. Considering the expected cumulative impact of various stressors, the medium level of burnout observed has come as a surprise to us and might actually be considered as relatively good news. Nevertheless, no level of burnout is negligible and has wide ranging negative consequences.
\end{abstract}

Keywords: Burnout, caregivers, COVID-19, geriatric facilities

\footnotetext{
*Correspondence to: Mohamad El Haj, Faculté de Psychologie, LPPL - Laboratoire de Psychologie des Pays de la Loire, Univer-
} 


\section{INTRODUCTION}

COVID-19 is a disease caused by the novel coronavirus SARS-CoV-2 that primarily affects the respiratory tract and produces clinical symptoms, including dry cough, dyspnea, fever, and potentially serious lung damage and death. Despite the rigorous implementation of global social-distancing measures and quarantine, the incidence of COVID-19 continues to rise. While some patients infected with COVID-19 develop only mild symptoms and may spontaneously recover, other patients may develop fatal complications including severe pneumonia, pulmonary edema, acute respiratory distress syndrome, and organ failure [1]. Notably, patients infected with COVID-19 requiring intensive care support have been mostly elderly individuals with multiple comorbidities, including cardiovascular, endocrine, digestive, and respiratory diseases [2]. The consequences of the COVID-19 pandemic have been prominent for elderly individuals receiving acute and subacute care in geriatric facilities. In its effort to limit the spread of COVID-19, the France government has instructed Hospitals and geriatric facilities to restrict, from 15 March 2020 onward, all visitations except for end of life situations. Nursing homes have even been instructed to restrict all activities considered as non-essential, including services by non-essential healthcare personnel (e.g., hairdressers), group activities, and even communal dining. In some cases, activities occurring outside residents' rooms have even been restricted. These measures, as implemented during the advanced phases of fight against COVID-19 in France, do not only impact the residents, but also the healthcare workers who, besides their usual duties, are asked to demonstrate increased vigilance and perform additional medical duties, such as regularly monitoring residents for potential fever and respiratory symptoms. Moreover, the resulting deaths have led to increased postmortem care. Other challenges for healthcare workers include dealing with shortages in equipment and supplies, including sanitizers, gloves, and facemasks. Staffing shortages also add to the healthcare workers' burden, as some of their colleagues regularly become sick and others are found to be positive for the virus and are asked to remain quarantined in their homes to avoid contaminating others, but still remain unable to see their own families. Lastly, healthcare workers face challenges regarding safeguarding their own health and safety and those of their families. In order to help understand the mental health consequences of all these challenges, we assessed levels of burnout in caregivers in geriatric facilities in France during the COVID-19 crisis.

Burnout can be generally defined as a psychosomatic syndrome that arises in response to chronic and interpersonal stressors in the workplace [3, 4]. In geriatric facilities, caregiver burnout yields a state of physical, emotional, and mental exhaustion that may include depression, anxiety, and distress [5]. More specifically, burnout in geriatric facilities can be characterized by 1) emotional exhaustion, such as lack of energy and enthusiasm and decreased emotional resources, 2) reduction of personal fulfillment, such as perceiving care as negative or ineffective, and 3) depersonalization, such as loss of idealism and adoption of an indifferent or even cynical attitude toward residents [6]. Caregivers in geriatric facilities are at significant risk for burnout even at baseline, especially when working with residents with dementia that have functional and cognitive decline and challenging behavioral and psychiatric problems (e.g., hallucinations) [7] that can lead to violence [8]. Working with patients with dementia also involves confrontation with serious illness and death $[9,10]$. Besides these largely unavoidable factors, organizational factors (e.g., rates of employee turnover in the institutions) may significantly influence the dynamics of burnout [11]. A growing attention to caregiver's burnout in geriatric facilities has stemmed from concerns over caregivers' wellbeing as well as the risks of worsening professional performance, absenteeism, and consequent work stoppages.

Consequently, burnout in healthcare workers in geriatric facilities is a major public health problem. We hypothesize that levels of burnout have increased due to the COVID-19 crisis and its consequences. Thus, it is critical to assess burnout in caregivers during the COVID-19 crisis. We tackled this challenge by inviting caregivers in France to fill an online survey during the COVID-19 crisis; the survey was an online version of the Oldenburg Burnout Inventory (OLBI) [12], a scale assessing burnout in terms of affective, cognitive, and physical exhaustion and disengagement. More specifically, this scale consists of 16 positively and negatively formulated items that evaluate exhaustion and disengagement. These items mirror the assumption that exhaustion and disengagement can be interpreted in terms of a continuum that ranges from disengagement to dedication (i.e., the identification continuum) and a continuum that ranges from exhaustion to vigor (i.e., the energy continuum) [12]. Regardless of these 
theoretical considerations, we chose the OLBI, as this instrument has been validated in several occupational spheres [13]. Research has also demonstrated the psychometrical robustness of this scale [14-16]. A French version of this scale has also been validated [17]. Critically, with 16 items, the OLBI is relatively short so caregivers needed to only devote a short lapse of their valuable time to complete the survey.

Together, the COVID-19 crisis is increasing the burden of caregivers in geriatric facilities. In order to evaluate whether this crisis is leading to burnout in caregivers, we assessed, using an online survey based on the OLBI scale, burnout among caregivers during the COVID-19 crisis. We assessed the total burnout scores of caregivers on the OLBI scale, and more specifically, their scores regarding the two dimensions of the scale (i.e., exhaustion and disengagement). We expected high levels of disengagement, exhaustion, and overall burnout in caregivers. These levels can be expected in light of the fatigue, loss of energy, and/or feelings of being emotionally overextended and exhausted by the work, as may be experienced by the caregivers during the COVID-19 crisis.

\section{METHODS}

\section{Participants}

The study sample included 84 participants (59 women, 25 men, $M$ age $=32$ years, $S D=10.32$ ). Thirty-three participants were nursing assistants, twenty-five were nurses, fifteen were physicians, and eleven were service/environment agents (agents charged of maintaining hygiene levels). All declared working in geriatric facilities in France.

\section{Procedures}

We established an online version, with Limesurvey, of the validated paper-and-pencil French version of OLBI [17]. We directly distributed the online survey, by emails or personal invitations, to hospital healthcare workers working in the Hospitals of Nantes, Lille, and Angers. We also distributed the online survey by emails to colleagues working in other hospitals. The online survey was also distributed through social networks (e.g., Facebook groups of geriatric caregivers). The survey began on April 12, 2020, and ended on April 24, 2020.

The survey opened with a message stating that the aim of the study was to assess the participants' work experience during the COVID-19 crisis. Next, the survey provided an informed consent form explaining anonymity, confidentiality, and that the purpose of the study was solely scientific. Participants were provided with the contact details of the first author, for any queries. Once participants consented to participate, they were provided with sociodemographic questions on sex, age, and profession. Next, participants were presented with the 16 items of the OLBI scale, provided in Table 1. Half of the items assessed the exhaustion dimension of burnout, whereas the other half measured the disengagement dimension of burnout. Half of the items were positively worded, whereas the other half were negatively worded. Participants were invited to respond to each item by using a scale ranging from 1 (strongly agree) to 4 (strongly disagree). When analyzing the data, we reversed scores on negatively worded items so all high scores would refer to high levels of exhaustion and disengagement. Thus, the maximum score on each one of the exhaustion and disengagement subscales was 32 points ( 8 items $\times 4$ points for each subscale) and the total score for burnout was 64 points.

Following the French validation and scoring system of the OLBI scale, scores can be considered as indicating:

- Low exhaustion for scores $<16 / 32$ points

- Medium exhaustion for scores between 16 and $23 / 32$ points

- High exhaustion for scores $>23 / 32$ points.

- Low disengagement for scores $<15 / 32$ points

- Medium disengagement for scores between 15 and $22 / 32$ points

- High disengagement for scores $>22 / 32$ points.

- Low burnout for scores $<30 / 64$ points

- Medium burnout for scores between 30 and 44 /64 points

- High burnout for scores $>44$ /64 points.

\section{RESULTS}

Scores on the exhaustion and disengagement subscales and the total scale are provided in Table 2 . To compare scores to the validated cut-offs we used non-parametric tests (i.e., Wilcoxon signed rank tests) owing to the scale nature of the variables and their abnormal distribution. We provided effect size ( $d=0.2$ can be considered a small effect size, $d=0.5$ represents a medium effect size and $d=0.8$ refers to a large effect size [18]). The effect size was calculated for non-parametric tests following recommendations 
Table 1

Oldenburg Burnout Inventory

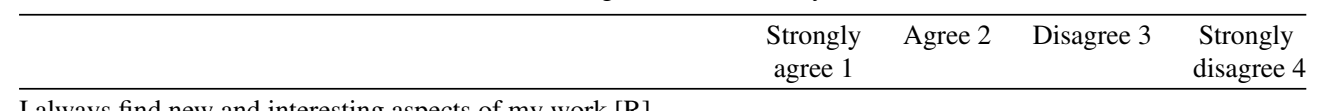

I always find new and interesting aspects of my work [R]

There are days when I feel tired before work

It happens more and more often that I talk about my work in a negative way

After work, I tend to need more time than in the past in order to relax and feel better

I can tolerate the pressure of my work well [R]

Lately, I tend to think less at work and do my job automatically

I find my work to be a positive challenge [R]

During work, I often feel emotionally drained

Over time, one can become disconnected from this type of work

After work, I have enough time for my leisure activities [R]

Sometimes I feel sickened by my work tasks

After my work, I usually feel worn out and weary

This is the only type of work I can imagine myself doing [R]

Usually, I can manage the amount of my work well [R]

I feel more and more engaged in my work [R]

When I work, I usually feel energized [R]

In statements marked [R] reverse coding was used; a negative response indicates high burnout and a positive answer indicates low burnout. Disengagement items are 1, 3, 6, 7, 9, 11, 13, and 15. Exhaustion items are 2, 4, 6, 8, 10, 12, 14 , and 16 .

Table 2

Mean scores on the exhaustion and disengagement subscales of the Oldenburg Burnout Inventory scale

\begin{tabular}{lcc}
\hline Exhaustion & Disengagement & Total \\
\hline $20.94(5.68)$ & $17.40(4.89)$ & 38.35 \\
\hline
\end{tabular}

Standard deviations are given between parentheses; the maximum score on each subscale was 32 points; the maximum total score (exhaustion + disengagement subscales) was 64 points.

by Rosenthal and DiMatteo [19], and Ellis [20]. For all tests, level of significance was set as $p \leq 0.05$.

\section{Mean exhaustion, disengagement, and burnout in caregivers}

Analysis showed that the mean score for exhaustion, as provided in Table 2, was significantly higher form the cutoff value of $16(Z=6.13, p<0.001$, Cohen's $d=1.80$ ) and lower from the cutoff value of $23(Z=2.94, p=0.003$, Cohen's $d=0.68)$. Thus, this mean score indicates medium level of exhaustion. Regarding disengagement, the mean score was significantly higher form the cutoff value of $15(Z=3.94$, $p<0.001$, Cohen's $d=0.95)$ and lower from the cutoff value of $22(Z=6.29, p<0.001$, Cohen's $d=1.88)$. Thus, this score indicates medium disengagement. Regarding burnout, the total score was significantly higher form the cutoff value of $30(Z=6.58, p<0.001$, Cohen's $d=2.06$ ) and lower from the cutoff value of
$44(Z=5.02, p<0.001$, Cohen's $d=1.31)$, indicating medium burnout.

\section{Complementary analysis}

We compared burnout scores between the professional categories of participants [(i.e., nursing assistants: $M=37.66, \mathrm{SD}=(3.32)$, nurses: $M=38.89$, $\mathrm{SD}=$ (3.32), physicians: $M=37.21, \mathrm{SD}=$ (3.19), service agents: $M=39.42, \mathrm{SD}=(3.76)]$ and found no significant differences between these categories $\left[\chi^{2}\right.$ $(1, N=84)=0.36, p=0.55]$.

\section{DISCUSSION}

The COVID-19 crisis is significantly increasing the burden of healthcare workers in geriatric facilities providing acute and subacute care. We therefore investigated whether this crisis is leading to burnout in hospital healthcare workers. Contrary to our a priori hypothesis, analysis of their responses showed medium levels of disengagement, exhaustion, and overall burnout.

Medium levels of exhaustion, as observed in caregivers, can reflect fatigue, loss of energy, and/or feelings of being emotionally overextended and exhausted by the work, which are commonly being described by healthcare workers as being "empty", "worn out", or "feeling used up at the end of the shift". In addition, participants in our study have 
experienced medium levels of disengagement, which reflects a tendency to distance themselves from the object and the content of their work and/or negative attitudes toward their work. Since the number of patients' deaths has increased during the COVID-19 crisis in geriatric facilities, staff has been exposed to an increased number of traumatic events. This added stress may be expected to induce burnout [21], and may account for the levels of exhaustion and disengagement reported in our sample.

In our view, although our study demonstrates the presence of medium levels of healthcare worker burnout, this comes as a somewhat positive surprise, since we have expected higher levels of exhaustion, disengagement, and burnout after several weeks of dealing with the COVID-19 crisis. Healthcare workers are currently dealing with significant changes in the practices in their institutions, frequently longer shifts, increased demands for performing medical duties or postmortem care, shortage in equipment and supplies, staffing shortages and concerns related to their own safety and that of their families. In addition, healthcare workers are dealing with a novel disease for which they have never been adequately trained or prepared. Considering the expected cumulative impact of various stressors, the medium level of burnout observed has come as a surprise to us and might actually be considered as relatively good news.

Form an institutional perspective, the mean burnout among healthcare workers in our study can be attributed to organizational and institutional factors that may play a significant role in the genesis of burnout, following the social model of stress [22]. A geriatric facility (e.g., retirement home, nursing home, geriatric medical unit) is always integrated into a global health system. Thus, the expectations of the general public and public and private sector employers can determine the burnout experienced by healthcare workers. In our view, these variables can influence the sense of ownership of care (i.e., whether caregivers consider themselves as involved in the care they provide or partake in institutional decisions regarding COVID-19). This sense can be further influenced by trade power considerations (e.g., job insecurity for temporal agents) and human resource policies on contracting, opportunities for career development and remuneration. Thus, besides the increased workload directly resulting from the COVID-19 crisis, burnout among caregivers might be partially attributed to institutional or even broader societal factors (e.g., widespread economic insecurity).
While we tend to offer a positive view of the medium levels of burnout as reported by caregivers in this study, this is by no means negligible. Any level of burnout can cause serious psychological consequences, such as low self-esteem, anxiety, and frustration, as well physiological consequences, such as insomnia, headaches, pain, and even gastrointestinal problems, or lead to consumption of caffeine or tranquilizers and drugs [23, 24]. These serious consequences can impact the mental and physical health of caregivers, as well as the quality of care they provide. It is thus important to consider strategies to decrease the burnout in caregivers during and after the COVID-19 crisis. During the crisis, we recommend that institutions should enable caregivers to have brief intervals, even for few minutes, to allow them to communication with friends and families or to engage in mindfulness. In a related vein, mindfulness-based therapy should be provided once the crisis has passed. There is a large body of research suggesting positive effects of mindfulnessbased therapy on burnout, as this therapy allows for acknowledgement and adaptation of emotional and behavioral responses and development of problemfocused strategies rather than avoidance behaviors [25-28].

Geriatric facilities and extended care institutions are conceived and advertised as safe havens for the elderly, places where they may feel safe, "at home", and where they can expect to receive quality care. For healthcare workers, geriatric facilities are also conceived and advertised as places that allow them to provide high quality care, consequently, experience fulfillment. Yet, the COVID-19 crisis has impacted the sense of wellbeing experienced by both patients and caregivers. We are hopeful that, by offering the first empirical evidence on burnout in caregivers in geriatric facilities, our report will pave the way for remediation strategies to benefit those on the frontlines of the COVID-19 crisis.

\section{ACKNOWLEDGMENTS}

The authors would like to thank the caregivers who, despite the increased workload during the COVIDcrisis, have dedicated their time to answer the survey. Dr. El Haj was supported by the LABEX (excellence laboratory, program investment for the future) DISTALZ (Development of Innovative Strategies for a Transdisciplinary Approach to Alzheimer Disease) and the EU Interreg CASCADE 2 Seas Programme 
2014-2020 (co-funded by the European Regional Development Fund). This research was supported in part (for author DK) by the Intramural Research Program of the National Institute on Aging, NIH.

Authors' disclosures available online (https:// www.j-alz.com/manuscript-disclosures/20-1029r1).

\section{REFERENCES}

[1] Sohrabi C, Alsafi Z, O'Neill N, Khan M, Kerwan A, Al-Jabir A, Iosifidis C, Agha R (2020) World Health Organization declares global emergency: A review of the 2019 novel coronavirus (COVID-19). Int J Surg 76, 71-76.

[2] Wang D, Hu B, Hu C, Zhu F, Liu X, Zhang J, Wang B, Xiang H, Cheng Z, Xiong Y, Zhao Y, Li Y, Wang X, Peng Z (2020) Clinical characteristics of 138 hospitalized patients with 2019 novel coronavirus-infected pneumonia in Wuhan, China. JAMA 323, 1061-1069.

[3] Freudenberger HJ (1974) Staff burn-out. J Soc Issues 30, 159-165.

[4] Maslach C, Jackson SE (1981) The measurement of experienced burnout. J Organ Behav 2, 99-113.

[5] Yu H, Wang X, He R, Liang R, Zhou L (2015) Measuring the caregiver burden of caring for community-residing people with Alzheimer's disease. PLoS One 10, e0132168.

[6] Yildizhan E, Oren N, Erdogan A, Bal F (2019) The burden of care and burnout in individuals caring for patients with Alzheimer's disease. Community Ment Health J 55, 304310.

[7] El Haj M, Roche J, Jardri R, Kapogiannis D, Gallouj K, Antoine P (2017) Clinical and neurocognitive aspects of hallucinations in Alzheimer's disease. Neurosci Biobehav $\operatorname{Rev}$ 83, 713-720.

[8] Le Borgne M, Boudoukha AH, Garnier P-H, Jeoffrion C, Capponi I (2015) Inpatients-to-caregivers violence in geriatric settings: Impact of assaults on caregivers' mental health, risk, and protective factors. Traumatology 21, 259-266.

[9] El Haj M, Kapogiannis D, Antoine P (2020) The (fatalistic) present as experienced by individuals with Alzheimer's disease: A preliminary study. Neurol Sci 41, 427-433.

[10] El Haj M, Antoine P (2016) Death preparation and boredom reduction as functions of reminiscence in Alzheimer's disease. J Alzheimers Dis 54, 515-523.

[11] Estryn-Béhar M, Van der Heijden BIJM, Ogińska H, Camerino D, Le Nézet O, Conway PM, Fry C, Hasselhorn H-M (2007) The impact of social work environment, teamwork characteristics, burnout, and personal factors upon intent to leave among European Nurses. Med Care 45, 939950.

[12] Demerouti E, Nachreiner F (1998) Zur Spezifität von burnout für dienstleistungsberufe: Fakt oder artefakt [The specificity of burnout for human services: Fact or artefact]. Z Arbeitswiss 52, 82-89.
[13] Reis D, Xanthopoulou D, Tsaousis I (2015) Measuring job and academic burnout with the Oldenburg Burnout Inventory (OLBI): Factorial invariance across samples and countries. Burnout Res 2, 8-18.

[14] Halbesleben JRB, Demerouti E (2005) The construct validity of an alternative measure of burnout: Investigating the English translation of the Oldenburg Burnout Inventory. Work Stress 19, 208-220.

[15] Halbesleben JRB (2010) The role of exhaustion and workarounds in predicting occupational injuries: A crosslagged panel study of health care professionals. J Occup Health Psychol 15, 1-16.

[16] Timms C, Brough P, Graham D (2012) Burnt-out but engaged: The co-existence of psychological burnout and engagement. J Educ Adm 50, 327-345.

[17] Angenot A, Hanzez I (2013) Structural confirmation of the French version of the Oldenburg Burnout Inventory (OLBI). European Association of Work and Organizational Psychology. http://hdl.handle.net/2268/149404

[18] Cohen J (1988) Statistical power analysis for the behavioral sciences, Erlbaum Associates, Hillsdale, NJ.

[19] Rosenthal R, DiMatteo MR (2001) Meta-analysis: Recent developments in quantitative methods for literature reviews. Annu Rev Psychol 52, 59-82.

[20] Ellis PD (2010) The Essential Guide to Effect Sizes: Statistical Power, Meta-Analysis, and the Interpretation of Research Results., Cambridge University Press, New York, NY.

[21] Boudoukha AH, Altintas E, Rusinek S, Fantini-Hauwel C, Hautekeete M (2013) Inmates-to-staff assaults, PTSD and burnout: Profiles of risk and vulnerability. J Interpers Violence 28, 2332-2350.

[22] Hansen N, Sverke M, Naswall K (2009) Predicting nurse burnout from demands and resources in three acute care hospitals under different forms of ownership: A cross-sectional questionnaire survey. Int J Nurs Stud 46, 95-106.

[23] Cherniss C (1992) Long-term consequences of burnout: An exploratory study. J Organ Behav 13, 1-11.

[24] Weber A, Jaekel-Reinhard A (2000) Burnout syndrome: A disease of modern societies? Occup Med (Lond) 50, 512517.

[25] Cohen-Katz J, Wiley S, Capuano T, Baker DM, Deitrick L, Shapiro S (2005) The effects of mindfulness-based stress reduction on nurse stress and burnout: A qualitative and quantitative study, part III. Holist Nurs Pract 19, 78-86.

[26] Goodman MJ, Schorling JB (2012) A mindfulness course decreases burnout and improves well-being among healthcare providers. Int J Psychiatry Med 43, 119-128.

[27] Irving JA, Dobkin PL, Park J (2009) Cultivating mindfulness in health care professionals: A review of empirical studies of mindfulness-based stress reduction (MBSR). Complement Ther Clin Pract 15, 61-66.

[28] Singh NN, Lancioni GE, Karazsia BT, Chan J, Winton AS (2016) Effectiveness of caregiver training in MindfulnessBased Positive Behavior Support (MBPBS) vs. Training-asUsual (TAU): A randomized controlled trial. Front Psychol 7, 1549 . 